\title{
Información sobre Planificación Familiar que recibieron las adolescentes después de un evento obstétrico
}

\section{Family Planning Information on receiving the teenagers after an obstetric event.}

Mtra Catalina Reséndiz Flores • Mc. María Cristina Martha Reyes ••

Dra. en Enfermería María Teresa Cuatmatzi Peña..•

\section{Resumen}

Introducción: la información sobre Planificación Familiar se orienta hacia la salud reproductiva de las adolescentes, ejerciendo derecho a decidir libre, responsable e informada sobre el número y espaciamiento de sus hijos. Objetivo: Identificar la información sobre planificación familiar que recibieron las adolescentes después de un evento obstétrico por las enfermeras, en los servicios de Tococirugía y Ginecobstricia. Metodología: Estudio descriptivo transversal, sustentado en el modelo de Dorothy E. Johnson, participaron 30 adolescentes de 15 a 19 años de edad atendidas en el Hospital General de Coyuca de Catalán, Guerrero. Losdatos se recolectaron con un instrumento estructurado de 20 preguntas cerradas, validado con alfa de Cronbach de 0.87. Resultados: El 70\% de las adolescentes aceptaron más la información verbal y demostrativa, el 26\% selecciono el Dispositivo Intrauterino, el $16.7 \%$ prefirió el condón decisión en pareja, el $13 \%$ resolvió utilizar inyectables con progestina, un 10\% progestágenos con estrógenos, se encontró una asociación estadística entre la edad y el nivel escolar lo que permitió la aceptación de la información y la elección de los métodos anticonceptivos. Discusión: Según Millán las diferencias culturales, educativas y otros factores inciden grandemente en la capacidad de comprensión de las usuarias en Planificación Familiar. Conclusión: la información proporcionada por las enfermeras en los servicios de Tococirugía y Obstetricia influyó en las adolescentes para las decisiones que tomaron.

- Profesora de la Universidad Autónoma de Guerrero.

- Profesora de tiempo completo de la Facultad de Enfermería de la Universidad Michoaacana de San Nicolás de Hidalgo, Morelia Michoacán.

... Profesora de tiempo completo de la facultad de Estudios Superiores Zaragoza UNAM.

Correspondencia • Mtra Catalina Reséndiz Flores: careflor_06@yahoo.com.mx

RECIBIDO: 10 DE DIC DE 2009.

ENVIADO A CORRECIONES: 8 DE FEBRERO DE 2010.

ACEPTADO: 30 DE MARZO DE 2010 


\section{Key Words: \\ Family Planning, Information, teenager.}

Introductión: Information about Family Planning is geared toward teenagers reproductive health, exercising the right to decide freely, responsibly and informed on the number and spacing of their children. Objective: To identify the Family Planning information received after an event teenagers obstetric nurses, services and Ginecobstricia Tococirugía. Methodology: Descriptive study, based on the model of Dorothy E. Johnson, involved 30 teenagers from 15 to 19 years of age treated at the Hospital General de Coyuca de Catalán, Guerrero. The data were collected using a structured instrument of 20 closed questions, validated with Cronbach alpha of 0.87 . Results: $70 \%$ of teens agreed more verbal information and demonstration, $26 \%$ selected the Intrauterine Device, $16.7 \%$ preferred the condom decision as a couple, $13 \%$ decided to use injectable progestin, a progestin with estrogen $10 \%$, An association was found between age and educational level which allowed the acceptance of information and choice of contraceptive methods. Discussion: According to Millán cultural differences, education and other factors greatly affect the ability of understanding of users in Family Planning. Conclusion: The information provided by nurses in the Obstetrics and services Tococirugía teenagers influenced the decisions they made.

\section{INTRODUCCIÓN}

La información de Planificación Familiar es una estrategia educativa que busca la adopción de cambios voluntarios en la conducta de las adolescentes que experimentaron un parto, cesárea y aborto, sin olvidar las determinantes sociales y culturales. Donde en este escenario el profesional de enfermería informa sobre la Planificación Familiar, para prevenir los embarazos no planificados y espaciamiento de los mismos, considerando los derechos sexuales y reproductivos. ${ }^{1}$

En México en 1994 se fortaleció el Programa de Planificación Familiar alintegrarse el concepto holístico de la salud reproductiva, implementando un subcomponente de anticoncepción Posparto, transcesárea, poscesá- rea y Posaborto; este Programa se otorgó en las Unidades Medicas desde las décadas de los sesenta considerada como prioridad de atención dentro del Plan Nacional de salud. ${ }^{2}$ Como resultado de este avance en el Estado de Guerrero el impacto del Programa de Planificación Familiar ha sido evidente, principalmente por la aceptación que ha tenido la población adolescente. En las últimas tres décadas se ha notado un descenso en los niveles de fecundidad asociados a incrementos importantes en el uso de los métodos anticonceptivos; a principio de la década de los 70'S el promedio de hijos por mujer era superior a 8 , mientras que en la actualidad el numero esperado es cercano a 2.5, sin embargo en el Estado continua la alta prevalencia de morbilidad materna; la proporción de abatimiento de la tasa de fecundidad es 3 veces mayor a la $\mathrm{Na}$ cional en los últimos 30 años. ${ }^{3}$ En este discernimiento, es importante destacar que las adolescentes en periodos de posparto, poscesarea, posaborto, pretendan prevenir un embarazo no planificado o desean posponer o espaciar los nacimientos. ${ }^{4}$ Para coadyuvar es imprescindible como función principal que el profesional de enfermería promueva los métodos de Planificación Familiar, mediante la información y consejería considerada como una necesidad inmediata para las adolescentes, ya que con frecuencia la fertilidad regresa al cabo de dos semanas después del aborto y en el posparto generalmente no hay ovulación durante las primeras seis semanas. Por lo que la información 
en Planificación Familiar es fundamental como estrategia educativa sobre todo durante la atención prenatal y posterior a un evento obstétrico, ya que se ha comprobado que contribuye a disminuir la incidencia de embarazos de alto riesgo asociados a la reproducción en edades extremas, múltiparidad y presencia de factores de riesgos reproductivos. $^{5}$

Este estudio se refiere a lainformación de Planificación Familiar, para llevarlo a cabo fue con base al Modelo Teórico de Dorothy E. Johnson ya que considera al paciente como un Sistema Conductual del cual forma parte el subsistema sexual que se encarga de la procreación, dentro de su metaparadigma conceptualiza a enfermería como una fuerza reguladora externa que actúa para preservar la organización de la conducta del paciente cuando se siente sometido a un estrés. ${ }^{6} \mathrm{El}$ estrés manifestado por las adolescentes después de procrear un hijo o experimentar un aborto, aunado a estos cambios la incapacidad en la toma de decisiones sobre la elección de los métodos anticonceptivos.

En este contexto las adolescentes se encuentran en proceso de cambio y tensión influidos por factores internos y externos, en especial los órganos reproductivos de la mujer adolescente que durante el embarazo y posterior a un parto, cesárea o aborto sufren importantes modificaciones, incluyendo aquellas modificaciones del ciclo menstrual que depende de las hormonas ováricas estrógenos y progesterona, actuando sobre el endometrio y la función ovárica, originando un desequilibrio conductual ineficaz por todos estos cambios y alteraciones fisiológicas, psicológicas, y sociales. ${ }^{7}$ Enfermería según Johnson esta designada a ayudar a las personas para prevenir una enfermedad o lesión, de esta manera el profesional se transforma en un elemento de apoyo que ayuda a las adolescentes por medio de la información, ha desarrollar capacidades para afrontar sus decisiones de la mejor manera referentes a la planificación familiar posteriormente de haber cursado un evento obstétrico. Tomando en cuenta que la información que reciban las adolescentes logren aclarar sus dudas, y por lo consiguiente que se sientan confortables y seguras de si mismas. En un estudio realizado en Oaxaca (2007), se encontró que el profesional de enfermería notó un cambio en la actitud de las adolescentes después de recibir información de planificación familiar, ${ }^{8}$ actualmente los desafíos actuales y futuros de la profesionalización en enfermería plantean la necesidad de un nuevo proceso formativo basado en principios de excelencia idoneidad y calidad para lo que se requiere capacidad de análisis, toma de decisiones, y transmisión de información considerados necesarios para el desempeño de la profesión. ${ }^{9} \mathrm{Se}$ cree oportuno informar a las adolescentes de Planificación Familiar con los siguientes elementos:

1. Tratar bien a las adolecentes, el buen trato es la regla de oro para establecer un clima de confianza y respeto.

2. Interactuar: compartir las experiencias y facilitar un espacio humanizado y de esta forma crear un ambiente adecuado para otorgar información.

3. Adaptar la información: no debemos olvidar las diferencias culturales, educativas y otros factores que inciden en la capacidad de comprensión de las adolescentes.

4. Evitar el exceso de información.

5. Promover en las usuarias los métodos anticonceptivos, realizar exploración física, identificar factores de riesgos que contraindique el método de planificación familiar. ${ }^{10}$

El Objetivo del estudio fue identificar la información sobre Planificación Familiar que reciben las adolescentes después de un evento obstétrico en los servicios de Tococirugía y Gineco obstetricia por parte del personal de Enfermería en el Hospital General de Coyuca de Catalán, Guerrero.

\section{Metodología:}

El estudio fue de tipo Cuantitativo, Descriptivo y Transversal, previo consentimiento informado se estudiaron 30 adolescentes con edad entre 15 y 19 años que tuvieron un evento obstétrico durante el tiempo del estudio, La técnica de recolección de datos se efectúo en los meses de septiembre a diciembre del 2008 por medio de un instrumento estructurado con 20 ítems, considerando los aspectos sociodemográficos (edad, estado civil, nivel escolar, ocupación), antecedentes gineco-obstetricos (inicio de vida sexual activa y edad del primer embarazo, aborto, parto o cesárea y número de hijos), tipo de información recibida, (rotafolios, cartel, trípticos, folletos, información verbal y videos). Métodos utilizados de planificación familiar. La validez del contenido se efectuó 
por medio de 5 expertos del área de salud reproductiva y con amplia experiencia docente, la prueba de confiablidad arrojo un Alfa de Cronbach de 0.820, para el análisis de los resultados se utilizó estadística descriptiva y pruebas no paramétricas de acuerdo a la naturaleza de las variables, a través de Chi cuadrada se probó la significancia de las diferentes proporciones y la correlación de Pearson para conocer el grado de asociación de las variables. ${ }^{11}$

\section{Resultados}

Del total de adolescentes entrevistadas, el $50 \%$ se encontraron en el rango de edad de 18 a 19 años, así mismo el 43.3\% estudiaron preparatoria o equivalente, se observo una correlación significativa entre la edad y la escolaridad ( $\mathrm{r}=.511$, $\mathrm{p}<0.004)$. El 70\% de las adolescentes acepto la información de Planificación Familiar , es necesario hacer énfasis, que hoy los modelos educativos desde el nivel instrucción primaria hasta preparatoria se ha está dando mayor importancia a la salud reproductiva en las adolescentes, debido a que han ido en aumento los embarazos no planeados, los abortos y las enfermedades de transmisión sexual, así mismo es importante resaltar que los adolescentes con mayor edad tienen mayor probabilidad de elegir un método anticonceptivo. (Cuadro 1)

Con respecto a la elección de los Métodos anticonceptivos y la Información de Planificación Familiar que recibieron las adolescentes en los Servicios de Tococirugía y Gineco-obstetricia se encontró que el Dispositivo intrauterino fue el método de mayor prevalencía elegido por las adolescentes con un $26.7 \%$, seguido del Condón con el 16.7\%, los Inyectables con Progestina en un $13.3 \%$, Inyectables Estrógenos y Progestina un 10\%. En el mismo porcentaje se encuentran los hormonales orales, el Parche Norplant 3.3\%, solo 3 adolescentes no decidieron métodos anticon-

\section{CUADRO No. 1}

Coeficiente de Correlación de Pearson de Edad y Nivel Escolar de las adolescentes del Hospital General Regional de Coyuca de Catalán, Gro. Octubre 2008. Correlaciones

\begin{tabular}{|ll|l|l|}
\hline Variables & \multicolumn{1}{c|}{$\begin{array}{l}\text { Edad } \\
\text { del } \\
\text { Adolescente }\end{array}$} & $\begin{array}{l}\text { Nivel } \\
\text { Escolar }\end{array}$ \\
\begin{tabular}{ll|l} 
EDAD DEL \\
ADOLES-
\end{tabular} & $\begin{array}{l}\text { Correlación de Pearson } \\
\text { Sig. (bilateral) }\end{array}$ & 1 & .004 \\
CENTE & $\mathrm{N}$ & 30 & 30 \\
NIVEL ES- & Correlación de Pearson & $.511(* *)$ & 1 \\
COLAR & Sig. (bilateral) & .004 & 30 \\
\hline
\end{tabular}

FUENTE: Cuestionario aplicado a las adolescentes

después de un Evento Obstétrico

ceptivos, con riesgo de que ocurra un embarazo no planeado con periodos intergenésicos cortos. (Grafica No. 1)

Con relación al medio de información sobre planificación familiar que recibieron las adolescentes después de haber cursado un evento obstétrico (parto, cesárea o aborto) se observó que el $36.67 \%$ recibió información de la enfermera de manera directa en forma verbal, seguido de la información por medio de trípticos y folletos con un $26.67 \%$, así mismo el personal de enfermería proyectó videos siendo este un medio muy relevante por contener más evidencias de los métodos anticonceptivos, en un $20 \%$. Por último el $16.67 \%$ recibió información por medio de rotafolios, carteles y demostración. (Grafica No. 2)

En cuanto a la información de la Programación de las citas subsecuentes el $73.3 \%$ contestó que la enfermera les informo de la próxima cita, un $26.7 \%$ no fueron informadas de las citas subsecuentes, entre las adolescentes no citadas se encuentran las que no desearon métodos anticonceptivos y la otra proporción corresponde a las adolescentes que aceptaron Dispositivo intrauterino y el Parche Norplant, es primordial la información de estos dos últimos métodos, por que presentan algunas alteraciones sobre todo el Parche Nortplant puede ocasionar hematoma en el sitio de aplicación e infección local. (Grafica No.3)

\section{DISCUSIÓN}

El subsistema sexual de las adolescentes comprendido según el Modelo de Dorothy Jonhson, es aplicable a la práctica de enfermería por que permite analizar la reproduc- 

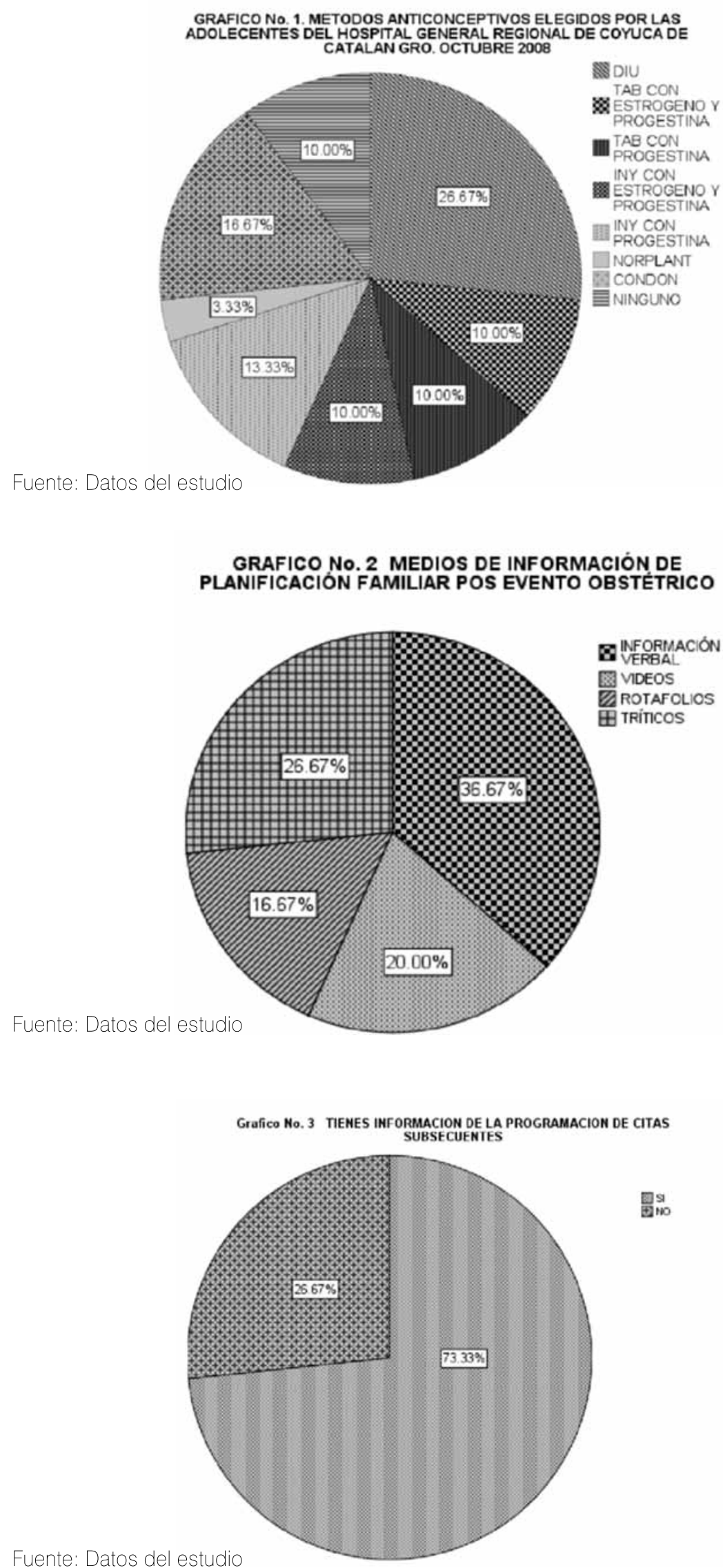

ción de las adolescentes versado en la Planificación Familiar después de un evento obstétrico, es fundamental que el profesional de enfermería actúe como una fuerza reguladora externa sin olvidar las características individuales, los factores del entorno y la forma de vida de las adolescentes.

La edad no es una limitación para usar un método anticonceptivo, ya que no hay razones biológicas que sustenten que una adolescente corra un riesgo mayor que una mujer de mayor edad. Según Herrera en su estudio, mostro que unos de los factores más frecuentes es el nivel educativo y la edad de las adolescentes, la mayoría tenia educación media superior seguido del nivel instrucción secundaria, son factores que intervienen considerablemente en la decisión de elegir un método de Planificación Familiar, ${ }^{12}$ puede atribuirse que la información fue de primera instancia otorgada en las escuelas sin embargo a pesar de adquirir información tuvieron embarazos en periodos intergenesicos cortos, la mayoría manifestó haber procreado de uno dos hijos. De acuerdo con Núñez, encontró que las dos terceras partes del total de las adolescentes tenían edades de 18 a 19 años y con nivel educativo preparatoria, recibieron información y eligieron métodos de planificación familiar, además la mayoría tenía información previa y la percepción en cuanto al servicio de Planificación Familiar era buena. ${ }^{13}$

El Dispositivo intrauterino y el condón fueron los anticonceptivos elegidos más frecuentes pudiera deberse a que solo se encuentran disponibles en los servicios de Tococirugia, en especial el dispositi- 
vo intrauterino que se inserta en el momento de la resolución del evento obstétrico, una de las ventajas de este método según Berek, es que no posee ningún efecto sobre la lactancia, es fundamental informar sobre las reacciones secundarias, cuando existen dudas se corre el riesgo de que se produzcan fallos con el método y puede llevar al abandono del mismo por miedo a comprometer su salud, o por el contrario no dar importancia a reacciones o síntomas secundarios. ${ }^{14}$ Estos resultados fueron similares al del Estado de Morelos según Núñez, sobre la utilización de métodos anticonceptivos, se mostró que la mayoría prefirió un método de Planificación Familiar, el más utilizado fue el Dispositivo Intrauterino, esto refleja la influencia del Programa en los hospitales donde el profesional de enfermería promueve su utilización.

\section{CONCLUSIÓN}

La información sobre Planificación Familiar que recibieron las adolescentes que cursaron un parto, cesárea y aborto, hospitalizadas en los servicios de Tococirugia y Gineco obstetricia se comprobó que la información otorgada por el profesional de enfermería, estuvo dirigida en los métodos anticonceptivos temporales y definitivos, el que más optaron fue el dispositivo intrauterino, seguido del condón masculino y los inyectables con progéstina. Se demostró que las adolescentes fueron informadas de las ventajas y efectos colaterales además la principal vía de información fue la oral, utilizaron material didáctico para la oferta de los métodos anticonceptivos. De acuerdo a los hallazgos se debe de reforzar la información a las adolescentes de menor edad y menor instrucción escolar, ya que el $30 \%$ de las adolescentes tenían edades de 15 años ubicadas en segundo lugar. Se sugiere implementar un Programa de información y consejería de Planificación Familiar después de un evento obstétrico dirigido a las /os adolescentes con horarios flexibles que les permita tener información eficaz, e incrementar las opciones de los métodos anticonceptivos.

\section{Referencias Bibliográficas}

1 Norma Oficial Mexicana NOM005-SSA2-1993 de los servicios de Planificación Familiar. Modificada 2004-México en Red. Recuperado el 14 de Mayo de 2009. Disponible:http//www.salud.gob. $\mathrm{mx} /$ unidades/cdi/nom//67ssa17. html

2 Domínguez GS. Consejería en Salud Sexual y Reproductiva para los adolescentes. Centro de Salud de la Micrfo red 3 de Febrero Pachaútec-Ventanilla Callao.AgostoOct.2007.

3 Secretaria de Salud del Estado de Guerrero. Sistema de Información en Salud. 2007, 12 pp.

4. Secretaria de Salud. Dirección General de salud Reproductiva, Anticoncepción, Posparto, Trancesárea, Poscesárea y Posaborto. México,2002

5 Mondragón-Castro, H. Obstetricia Básica e Ilustrada. 5ta. Edición. México. Edit. Trillas. 2008. p.134165.

6 Marriner-Tomey A. Modelos y Teorías en enfermería. 6ta. ed. España. edit. Elsevier Mosby. 2007. p. 130-142

7 Berek Jonathan S. Ginecología de Novak 13va. ed. México. Edit. McGraw-Hill Interamericana, 2008. p.191.
8 Billings D, González D, Ramírez R. Atención Posaborto en cuatro países de América Latina. 1er. ed. México. Edit. Ipas. 2007. p. 61.-73

9 Gutiérrez ML, Factor Determinante en el Ejercicio Profesional con Calidad en Enfermería. Rev. IMSS de México año 2008,16(3),121-125

10 Millán KT. Consejería Adolescente: Descripción epidemiológica y motivos de Consulta Rev. Médica Chile, año 2007,13 (5), 457-463.

11 Polit DF, Hungler BP. Investigación Científica en Ciencias de la Salud. 7ta. ed. México: McGraw-Hill Interamericana; 2006. P399-401

12 Herrera GV, Rodríguez DL, Quintero SM, Flebes TL. Anticonceptivo en la consulta de Planificación Familiar. Medicina General Integral, Cuba año 2007, 13(4) 345-351

13 Núñez UR, Hernández PB, García BC. González D. Embarazo no deseado en adolescentes y utilización de Métodos Anticonceptivos Posparto. Salud Publica de México, año 2003, 45(1) 92-102

14 Berek S. Ginecología de Novak 13va. ed. México. Edit. McGrawHill Interamericana, 2004. p.191.

\section{Nota}

**: La correlación es significativa al nivel 0,01 (bilateral). 